\title{
Prejuicios hacia las personas con discapacidad: fundamentación teórica para el diseño de una escala
}

Prejudices towards people with disability: theoretical foundation for designing a scale

\section{Volumen 20, Número 1 \\ Enero - Abril \\ pp. $1-26$}

\begin{abstract}
Alicia Gurdián-Fernández Marie-Claire Vargas Dengo Carmen Delgado Álvarez Andrés Sánchez Prada
\end{abstract}

\section{Citar este documento según modelo APA}

Gurdián-Fernández, Alicia; Vargas Dengo, Marie-Claire; Delgado Álvarez, Carmen y Sánchez Prada, Andrés. (2020). Prejuicios hacia las personas con discapacidad: fundamentación teórica para el diseño de una escala. Revista Actualidades Investigativas en Educación, 20(1), 1-26. Doi. 10.15517/aie.v20i1.40131 


\title{
Prejuicios hacia las personas con discapacidad: fundamentación teórica para el diseño de una escala
}

Prejudices towards people with disability: theoretical foundation for designing a scale

\author{
Alicia Gurdián-Fernández 1 \\ Marie-Claire Vargas Dengo ${ }^{2}$ \\ Carmen Delgado Álvarez ${ }^{3}$ \\ Andrés Sánchez Prada ${ }^{4}$
}

\begin{abstract}
Resumen: Nuestro propósito en este ensayo, en primer lugar, es presentar la fundamentación teórica para el diseño y elaboración de una escala de prejuicios hacia las personas con discapacidad que pueda utilizarse en contextos de educación superior. En segundo lugar destacamos cómo, a partir de una investigación cualitativa, utilizamos sus hallazgos a modo de pivote para construir una escala siguiendo una ruta eminentemente cuantitativa. Exponemos también el procedimiento seguido para obtener los primeros ítemes de la versión de la escala inicial que aún está en proceso de validación. Los prejuicios hacia las personas en condición de discapacidad constituyen el eje temático y la dimensión de género el sub eje. Nuestra posición argumentativa sostiene que los prejuicios hacia la discapacidad son socialmente construidos, están presentes en el ámbito universitario donde aparecen permeados por el género, por tanto es indispensable abordarlos si queremos promover una educación universitaria inclusiva. Las conclusiones giran en torno a la importancia de responder al aumento de la población con discapacidad que ingresa en las instituciones universitarias y cómo nuestra responsabilidad como investigadoras e investigadores debe hacer evidentes los componentes ético y político que están siempre presentes en toda investigación.
\end{abstract}

Palabras clave: prejuicio, discapacidad, género, universidad

\begin{abstract}
Our purpose in this essay, first of all, is to present the theoretical foundation for designing a prejudices scale towards people with disability. Second, we highlight how, based on qualitative research, we use its results as a pivot to build a scale following an eminently quantitative route. We also include the procedure followed to obtain the first items of the initial version of the scale which is still going through a validation process. Prejudices towards people with disability are the thematic axis and the gender dimension is a sub axis. Our argumentative posture maintains that prejudices towards people with disability is a social construction, as well as that prejudices exist within university context where are permeated by gender, therefore, it is indispensable to address them in order to promote an inclusive higher education. Conclusions point out the importance to respond to a growing population with disability within the university institutions and how the ethical and political components are always present in every research, and yet our responsibility as researchers should be to make them evident.
\end{abstract}

Key Words: prejudice, disability, gender, university

\begin{abstract}
${ }^{1}$ Catedrática-Investigadora Jubilada del INIE-Universidad de Costa Rica, Costa Rica. Investigadora Principal del Proyecto Construcción de una escala de prejuicios sutiles hacia la discapacidad. Dirección electrónica: agurdianf@gmail.com

${ }^{2}$ Catedrática-Investigadora de la Universidad Nacional (UNA), Costa Rica. Responsable en la UNA del Proyecto Construcción de una escala de prejuicios sutiles hacia la discapacidad. Dirección electrónica: marie.vargas.dengo@una.cr ORCID https://orcid.org/0000-0001-8468-9690

${ }^{3}$ Catedrática de Psicometría en la Facultad de Psicología de la Universidad Pontificia de Salamanca, España. Dirección electrónica: mcdelgadoal@upsa.es ORCID https://orcid.org/0000-0002-6757-6317

${ }^{4}$ Profesor Encargado de Cátedra de Diseños de Investigación e Intervención Sistémica en la Facultad de Psicología de la Universidad Pontificia de Salamanca, España. Dirección electrónica: asanchezpr@upsa.es ORCID https://orcid.org/0000-0002-6242-1236
\end{abstract}

Ensayo recibido: 28 de junio, 2019

Enviado a corrección: 01 de octubre, 2019

Aprobado: 18 de noviembre, 2019

Los contenidos de este artículo están bajo una licencia Creative Commons 


\section{Introducción}

El tema de las actitudes hacia las personas con discapacidad ha sido ampliamente investigado en distintos contextos de educación superior, indicando que las actitudes negativas hacia este colectivo son muy difíciles de cambiar (Hanafin, Shevlin, Kenny y Mc Neela, 2006; Lissi, Zuzulich, Salinas, Achiardi, Hojas y Pedrals, 2009). Sin embargo, los prejuicios -componente afectivo de las actitudes en algunos modelos teóricos- no lo han sido y continúan prevaleciendo en la sociedad y afectando la vida de muchas personas. En consecuencia, identificar los prejuicios sutiles hacia las personas con discapacidad fue uno de los detonantes que nos motivó a continuar investigando en este campo. El otro detonante fue el evidenciado en la tesis doctoral titulada: «Influencia de los prejuicios de un sector de la población universitaria respecto a la discapacidad en la construcción de una cultura institucional inclusiva. Universidad Nacional-Costa Rica, 2012-2013» en la que, mediante el análisis crítico del discurso en sus distintos niveles y dimensiones, Vargas (2013) logra identificar un conjunto de prejuicios sutiles y mostrar que los prejuicios sutiles hacia las personas con discapacidad prevalecen en la población académica universitaria costarricense.

Esto nos llevó a plantearnos, siguiendo a Platón, que el mundo -en el caso concreto el mundo universitario costarricense- no es en absoluto como parece: es decir, hay una diferencia significativa entre la apariencia y la realidad. $Y$ la mayoría de personas caemos en la trampa de confundir apariencia con realidad. La relación entre apariencia y realidad se convierte así en uno de los ejes estructurales de la reflexión sobre el ser y sobre lo real, por lo que la Alegoría de la Caverna de Platón pasa a ser una de las metáforas más fértiles sobre dicha reflexión filosófica: solo un arduo esfuerzo y un compromiso personal consciente nos permitirá a los seres humanos romper las cadenas de un mundo de sombras y apariencias para aproximarnos a la comprensión de lo real. Y nuestro deseo es que la población universitaria logre romper las cadenas del mundo de sombras en que les han sumido los prejuicios hacia las personas con discapacidad.

La realidad es un concepto polisémico que tiene múltiples aplicaciones en todas las áreas de pensamiento humano, tanto filosófico como científico, tecnológico, político, psicológico, sociológico y educativo entre otros; y de un modo paradigmáticamente revulsivo, en la Física en el último siglo. Históricamente, la definición de la realidad supone un problema filosófico. Determinar qué es real y qué no lo es ha sido motivo de análisis y debate 
durante toda la historia de la humanidad. Otra arista imposible de soslayar es el no poder afirmar que la realidad está formada sólo por cosas materiales, ya que en el campo de la física la frontera entre materia y energía ha dejado de ser nítida; y en el de la experiencia humana, las emociones y los sentimientos también son "reales" en el sentido que lo son las cogniciones.

Resulta válido plantearnos, entonces: si para la educación superior costarricense continúa siendo un desafío responder al aumento de la población con discapacidad que ingresa a las instituciones universitarias ¿Cómo superar las actitudes discriminatorias tanto dentro del aula como en el entorno universitario? ¿Cuál es la realidad cultural en cuanto a los prejuicios hacia las personas con discapacidad en las universidades públicas costarricenses? ¿Por qué es pertinente incorporar la dimensión de género en el estudio de las actitudes hacia las personas con condición de discapacidad en el ámbito educativo?

Ante dichos cuestionamientos es imprescindible señalar que los prejuicios sutiles se caracterizan porque: a) se relacionan con un sentimiento, juicio o preconcepción; b) no se expresan explícitamente; c) se contradicen en el discurso y la práctica; d) la persona que los tiene no los reconoce abiertamente; e) poseen una carga afectiva aparentemente favorable hacia la otra persona, pero su connotación es negativa ya que en ellos subyace una jerarquización; f) pueden provocar emociones negativas como rechazo, indiferencia o temor; g) marcan una diferencia entre la persona que los tiene y la otra persona a quien se le atribuye el juicio o sentimiento; h) revisten una apariencia benevolente: compasión, protección. Más aún, si aunado a lo anterior es común escuchar: "yo no tengo prejuicios", pues "no es políticamente correcto tenerlos".

\subsection{Antecedentes}

Un equipo de investigación constituido por especialistas en distintas áreas del conocimiento -una investigadora del Instituto de Investigación en Educación (INIE) de la Facultad de Educación de la Universidad de Costa Rica (UCR) y una del Centro de Investigación y Docencia en Educación (CIDE) de la Universidad Nacional (UNA), ambas universidades de Costa Rica; en coordinación con dos especialistas en Investigación Cuantitativa de la Facultad de Psicología de la Universidad Pontifica de Salamanca, Españapresentamos un proyecto de investigación sobre la "Construcción de una escala de prejuicios sutiles hacia las personas con discapacidad" para instituciones universitarias públicas de 
Costa Rica ${ }^{2}$, con el propósito de valorar y facilitar un proceso de toma de conciencia sobre los prejuicios hacia la discapacidad que subyacen, no sólo en el ámbito institucional, sino también en las emociones y sentimientos del estudiantado, profesorado y personal administrativo. Nuestra escala incluye además una sub-escala de género para valorar si el prejuicio opera de forma diferente en función del sexo de las personas con discapacidad.

Esta investigación tiene como propósito construir una escala que permita comprobar la presencia de prejuicios sutiles hacia las personas con condición de discapacidad en el ámbito de ambas universidades estatales costarricenses. Con un posicionamiento teórico en que la discapacidad es sin duda una construcción social (Marks, 1999; Priestly, 1998), las autoras y autor retomamos el concepto de prejuicio sutil (Pettigrew y Meertens, 2001) referido a un sentimiento o preconcepción generada sobre la persona con condición de discapacidad, sin evidencia certera de que su existencia sea real

Consideramos urgente abrir camino para identificar los prejuicios sutiles hacia las personas con discapacidad dada la pertinencia de la temática y porque además, no encontramos evidencias de estudios previos específicamente en este tema. Abordar dicha temática es un aporte a la sociedad costarricense en términos del contexto educación superior, dado que la población con discapacidad representa un desafío nacional y educativo pues hasta el día de hoy aún existen muchas actitudes prejuiciadas y discriminatorias en el ámbito universitario.

Los resultados obtenidos en el proyecto "Percepciones y realidades en la atención de las necesidades educativas: propuesta de desarrollo profesional y su implementación en la Universidad Nacional" (Fontana, Quirós y Vargas, 2015) señalan que el personal académico de la UNA posee construcciones conceptuales de la discapacidad desde una visión tradicional centrada en la deficiencia, entendida como ausencia, falta, alteración o carencia, lo cual influye en las actitudes y el trato hacia el estudiantado con discapacidad. Sin embargo, estas actitudes y prejuicios no siempre se identifican ni se reconocen como tales, a pesar de que continúan prevaleciendo en el imaginario colectivo en distintos sectores de la población.

Los prejuicios sutiles "permean la disposición de la población docente hacia los estudiantes con discapacidad que están matriculados en algunos cursos universitarios que

2 Proyecto de investigación inscrito en el INIE-UCR con código 724-B6-343, en el PRODIS-UCR con el código B643 y en la UNA con el código 0201-16. 
imparten" (Vargas, 2013, p.257). Por ejemplo, en algunas ocasiones cuando el personal académico se entera que una persona estudiante con algún tipo de discapacidad está inscrita en el curso que les corresponde impartir, sienten temor, preocupación e inseguridad sobre cómo enseñar y tratar a esa persona (Fontana, Quirós y Vargas, 2015) por desconocimiento, falta de preparación en este sentido o por actitudes y prejuicios que no se reconocen.

La oportunidad coyuntural y estructural de contar con una escala de esta naturaleza, radica en la importancia de disponer de un instrumento (escala) que permita diagnosticar, por ejemplo en una unidad académica universitaria, los prejuicios no manifiestos hacia las personas con discapacidad que subyacen en la población docente, administrativa y estudiantil a fin de proponer acciones, así como opciones curriculares y pedagógicas entre otras, que garanticen la construcción de una cultura universitaria inclusiva.

Luchar contra los prejuicios sutiles puede ser difícil; superarlos, aún más. La ventaja es que las personas que desean y están dispuestas a develarlos pueden lograrlo, siempre y cuando estén dispuestas a conocer cuáles son sus prejuicios y a llevarlos a un plano consciente para trabajarlos.

\section{Encuadre teórico-conceptual}

\subsection{El estudio de los prejuicios}

En la década de 1980, algunos autores como van Dijk (1984) se interesan en estudiar los prejuicios étnicos en relación con los contextos sociales en que se utiliza el lenguaje. Van Dijk (1984) sostiene que los prejuicios no son simplemente un fenómeno cognitivo sino social; destaca la importancia de la contextualización de los prejuicios, así como otros aspectos del enfoque social, como por ejemplo que los prejuicios se adquieren, se forman y se transforman en los procesos de socialización, interacción y comunicación. Van Dijk (1983) se refiere a los prejuicios como una clase específica de actitudes y los conceptualiza en términos de "sistemas organizados de creencias y opiniones alrededor de un centro socialmente relevante" (p. 196). Hace notar que los prejuicios son considerados 'erróneos' dado que existe la creencia de que 'no deben tenerse', razón por la cual muchas personas no admiten que los tienen.

Posteriormente, otros autores como Pettigrew y Meertens (2001) aportan al campo investigativo la conceptualización de prejuicios sutiles. Los prejuicios sutiles, de acuerdo con 
dichos autores, aluden a un sentimiento o preconcepción generada sobre otra persona sin evidencia certera de que su existencia sea real. Las personas no siempre reconocemos o aceptamos tener prejuicios sutiles, dado que son tácitos, contenidos, silenciosos y subyacen a lo que intencionalmente se expresa y deja ver. Según Pettigrew y Meertens (1997), si bien el prejuicio sutil está estrechamente relacionado con el prejuicio manifiesto, el prejuicio manifiesto se refiere a la forma tradicional en que ha sido entendido el prejuicio, mientras que el prejuicio sutil puede expresarse e identificarse por sí mismo, de manera separada del prejuicio manifiesto.

El sexismo ambivalente, según Glick y Fiske (1996), añade un aspecto fundamental que no incorpora el prejuicio en general: los sentimientos aparentemente positivos hacia el grupo socialmente desfavorecido de las mujeres; pero que bajo la actitud paternalista y protectora encubre la creencia de que se trata de alguien inferior que no puede ser, por tanto, tratado como igual. A este aspecto del prejuicio se le denominó "benevolente", frente al prejuicio clásicamente considerado como "manifiesto" u "hostil". El sexismo ambivalente subraya esta característica benevolente como más propia del llamado "nuevo sexismo", por su mayor aceptabilidad social.

Así pues, para Glick y Fiske (1996) el sexismo ambivalente está compuesto por dos componentes claramente diferenciados, aunque al mismo tiempo relacionados entre sí: el sexismo hostil y el sexismo benevolente El sexismo hostil coincide básicamente con el viejo sexismo. El sexismo benévolo se define como un conjunto de actitudes sexistas interrelacionadas hacia las mujeres, que las considera de manera estereotipada y limitadas a ciertos roles. Sin embargo, simultáneamente el sexismo benévolo según Montes Berges (2008):

Ambos tipos de sexismo (hostil y benévolo) tienen su origen en las condiciones biológicas y sociales comunes a todos los grupos humanos donde, por una parte, los hombres son los que poseen el control estructural de las instituciones económicas, legales y políticas, pero, por otra parte, la reproducción sexual proporciona a las mujeres otro tipo de poder: el poder diádico. Este se refiere al poder que procede de la dependencia en las relaciones entre dos personas, puesto que los hombres necesitan a las mujeres para la satisfacción de sus necesidades sexuales y la crianza de sus hijos. El poder diádico se refleja en casi todas las sociedades como diferentes formas de ideología: idealización de las mujeres como objetos amorosos, admirándolas por su 
rol como esposas y madres, y emitiendo conductas que denotan actitudes protectoras hacia ellas.

Puesto que el sexismo hostil y el sexismo benévolo se fundamentan en el rol de la identidad y de la sexualidad entre hombres y mujeres, es lógico prever que ambos tipos de sexismo estén positivamente correlacionados.(p.16)

Es esencial destacar que, según los autores citados por (Montes Berges, 2008), todos estos sentimientos positivos hacia las mujeres y las conductas que generan siguen siendo sexismo a pesar del tono afectuoso que puedan tener, por cuanto a) se realizan, no en virtud de las características de la persona sino debido a su pertenencia al grupo de las mujeres; y b) se inscriben en la dominación tradicional del hombre. "Es más, el sexismo benévolo puede llegar a ser más perjudicial que el sexismo hostil, puesto que puede utilizarse para compensar o legitimar el sexismo hostil" (2008, p. 16).

El sexismo benévolo posee tres constituyentes básicos de los que destacamos dos de los indicados, por la misma autora:

- La negación de la condición de igualdad para las mujeres, que deben ser tuteladas: "EI hombre debe cuidar y proteger a la mujer como si fuera un padre que cuida de sus hijos".

- La creencia de que las mujeres son diferentes a los hombres, connotando estas diferencias como aparentemente positivas, aunque con una funcionalidad ventajosa para los hombres; pues al definirlas como "complementarias" se les atribuye la función de aportar cuidados y otros soportes favorables para los hombres.

\subsection{Discapacidad y género}

La situación específica de las mujeres con condición de discapacidad (McD) ha recibido una atención insuficiente en los estudios sobre este problema, limitándose en la mayoría de los casos a aportar datos o describir su realidad, sin abordar el modo en que el género afecta los estereotipos y prejuicios sobre las Personas con condición de discapacidad (López, 2007). Los estudios feministas tampoco le han prestado suficiente atención en sus investigaciones. Existe por tanto un vacío que requiere, desde una perspectiva ética y emancipatoria, la incorporación de la dimensión de género al estudio de las actitudes hacia las personas con condición de discapacidad en el ámbito educativo (Delgado, 2015). La 
visibilización científica de las McD (mujeres con condición de discapacidad) requiere tanto de estudios que aborden su experiencia personal, como de investigaciones sobre el entorno que las estigmatiza doblemente al añadir a la discriminación por discapacidad, la discriminación de género.

Desde el modelo social de discapacidad -que sitúa sus orígenes en la propia sociedad organizada para gente sin discapacidad- resulta sumamente relevante incorporar la variable género en cuanto construcción social de la desigualdad a partir de la diferencia sexual (Amorós, 2005). El enfoque metodológico y las herramientas de investigación feminista (Harding, 1996), resultan por tanto útiles para abordar las diferencias de género en las actitudes hacia las Personas con condición de discapacidad. Si la investigación feminista ha puesto de relieve la relación entre la discriminación y la consideración cultural de la inferioridad de las mujeres (Saltzman, 1992), en el ámbito de la discapacidad se profundiza aún en mayor medida la jerarquización en función del género. El código patriarcal que genera la idea de superioridad masculina encuentra en el ámbito de la discapacidad una alianza estratégica para perpetuarse, combinando la devaluación de las Personas con condición de discapacidad con la devaluación de las mujeres (López, 2007). Los estereotipos tradicionales sobre la mujer ejercen un peso importante cuando se añade la discapacidad, profundizando así su invisibilidad y mermando sus posibilidades de realización personal, como lo han mostrado algunos estudios (García y Zarco, 2004).

Por lo anteriormente expuesto, para evitar el sesgo del androcentrismo en la investigación se hace necesario incorporar la dimensión de género en el estudio de las actitudes hacia las Personas con condición de discapacidad. El análisis de los sesgos de género en la investigación requiere revisar cuidadosa y críticamente los modelos teóricos de los que se parte, así como su trasfondo (Delgado, 2015; Ferrer y Bosch, 2005). Como bien afirma Ramírez-Morera (2015, p. 50):

Históricamente las sociedades se han desarrollado bajo la ideología del patriarcado que invisibiliza las diversidades sociales, entre ellas la de las mujeres, las cuales se encuentran mediatizadas por los preceptos masculinos que transversalizan nuestras etnias, opciones sexuales, grupos etarios, condiciones de discapacidad, socioeconómicas y locales, por lo tanto la influencia de las ideas androcéntricas se reproducen en las distintas instituciones de la sociedad civil; un ejemplo de ello son las entidades de educación superior, las cuales omiten la incorporación de las 
perspectivas de género y discapacidad en los currículos universitarios, cuyos resultados son la violencia estructural y simbólica.

En el estudio de prejuicio hacia las personas con discapacidad, se hace necesario visibilizar estructuras de dominación que inciden en el desplazamiento de ciertos grupos de mujeres del discurso y la práctica feminista para "incorporar el entrecruce de estrategias de dominación, así como el análisis de la forma en que el poder implica las subjetividades de los sujetos involucrados" (Suárez, 2011, p.48). Considerar los aportes de la teoría feminista en el estudio de la interseccionalidad de la opresión de las mujeres, y los debates teóricos que esta cuestión generó, supera con mucho los objetivos de este trabajo; pero resulta pertinente reseñar que los múltiples ejes de opresión de las mujeres (etnia, clase, nacionalidad, sexualidad, condición, etc.), han sido objeto de análisis por parte de los feminismos de la llamada tercera ola. En síntesis, se puede concluir como señala Chandra Mohanty (2011), que la multiplicidad de opresiones que sufren las mujeres en su diversidad, están conectadas con la opresión universal de género: "las diferencias y particularidades no son nunca simplemente "diferencias"; al conocer las diferencias y particularidades, podemos ver mejor las conexiones y los elementos comunes" (p.416).

\subsection{El análisis del discurso como estrategia}

Según sustenta Fairclough (1992), existe una gran cantidad de enfoques de análisis crítico del discurso, dentro de los cuales su foco de interés es el análisis relativo a los procesos de transformación social. En este campo se destacan autores como Iñiguez (2006) y van Dijk (1983), además de Fairclough (1992). Según Morales (2004), el trabajo de los autores mencionados ha influenciado a los analistas estadounidenses del discurso a profundizar en las relaciones ideológicas del mismo.

Incurrir en el análisis crítico del discurso consiste en develar las ideologías subyacentes al discurso y a las opiniones y juicios que se expresan. Se trata entonces de analizar cómo los discursos reflejan las creencias que las personas adoptan según los grupos a los que pertenecen. En palabras de van Dijk (1996, p. 23), se "intenta especificar qué expresiones o significados del discurso dan lugar a qué clase de inferencias u otros procesos mentales". Para ello, identifica distintas estrategias semánticas discursivas subyacentes importantes y posibles de develar en el análisis ideológico del discurso. Entre estas se encuentran: a) la estrategia de representación positiva de la persona que emite el 
discurso y de representación negativa de las otras personas, b) la presentación positiva del grupo de pertenencia y de rechazo del atributo negativo al referirse a las demás personas (denegaciones), c) enfatizar (acentuar) la información relacionada al grupo de pertenencia y desdibujar (descalificar) la información relativa al grupo ajeno.

La utilización de las estrategias mencionadas, por lo general, implica una valoración positiva del grupo de pertenencia (endogrupo) y una descalificación o valoración negativa del grupo al que no se pertenece (exogrupo), lo cual muchas veces se pone de manifiesto en la construcción de un argumento discursivo que combina ambas valoraciones, expresando la valoración positiva en la primera parte del argumento y la valoración negativa en la segunda parte de éste.

El análisis del discurso como proceso interpretativo de significados y estructuras discursivas, toma en cuenta el contexto en el que el discurso se da para poder interpretarlo a partir de la situación social y de las propiedades de las situaciones sociales que lo enmarcan. Es decir, que de alguna forma el discurso está sujeto al contexto y, por tanto, debe interpretarse dentro de éste.

A partir de la conceptualización de Pettigrew y Meertens (2001) del prejuicio sutil como sentimiento o preconcepción negativa, sin evidencia certera de que su existencia sea real, enriquecimos el constructo adoptando la formulación de Glick y Fiske (1996; 2001) para el sexismo, quienes consideran el prejuicio como un fenómeno ambivalente que incluye dos dimensiones:

- Hostil: incluye los aspectos "negativos" del prejuicio en términos de minusvaloración y discapacidad, resaltando los aspectos limitantes y deficitarios de las Personas con condición de discapacidad y la generalización de los mismos. Incluye las actitudes y conductas discriminatorias basadas en la supuesta inferioridad y se manifestaría como "proteccionismo dominante" (forma de control y subordinación) y/o "diferenciación competitiva" (representación de las Personas con condición de discapacidad como incompetentes).

- Benevolente: incluye los aspectos del prejuicio en términos de visión estereotipada asociada a capacidades restringidas de las Personas con condición de discapacidad, pero expresados en tono positivo, aparentemente no prejuiciosa, con actitudesconductas de "proteccionismo idealizado" (idealización "buenista"-ingenua de las 
Personas con condición de discapacidad). Resalta la fragilidad-debilidad y la necesidad de cuidado y protección.

Esta doble dimensionalidad se aplicó al sexismo con el término "ambivalente" y podría adaptarse para la discapacidad, ya que ambas dimensiones expresan una concepción "subordinada" de las personas con condición de discapacidad al tomar la discapacidad como el rasgo definitorio de la persona. La discapacidad actúa entonces como "marcador", dando lugar a una representación exogrupal que posibilita el prejuicio. Ambos aspectos pueden convivir en el prejuicio y la forma benevolente puede también enmascarar la forma hostil, por efecto de la deseabilidad social.

Partimos de la premisa de que los seres humanos tenemos prejuicios hacia las personas con condición de discapacidad; definimos como constructo central los prejuicios sutiles en tanto que son aquellos que subyacen a lo que intencionalmente se expresa Pettigrew y Meertens (2001), se relacionan con un sentimiento, juicio o preconcepción y por lo general tienen connotación negativa Vargas (2013). Los prejuicios sutiles hacia las PcD se caracterizan por hacer énfasis en la diferencia, en la categorización como exogrupo o sentido de "no pertenencia", el resentimiento por la discriminación positiva, la poca familiaridad, la falta de información y el desconocimiento y falta de información y el efecto perceptual o percepción selectiva a partir de la condición de discapacidad. También definimos los siguientes constructos de base y los relacionamos con categorías previamente definidas a partir de nuestro marco teórico referencial:

- Discriminación: es la manifestación externa del prejuicio, 'componente comportamental del prejuicio' (Montes Berges, 2008); el tratamiento desigual que desfavorece a un sujeto por su pertenencia a un exogrupo.

- Discapacidad: condición (motora/física, cognitiva/intelectual, sensorial) que se da en términos relacionales en la interacción con el entorno (Convención de sobre los Derechos de las Personas con Discapacidad del año 2006, ratificada por la Asamblea Legislativa de Costa Rica, 2008).

- Personas con condición de discapacidad PccD:

“...incluyen aquellas que tengan deficiencias físicas, mentales, intelectuales 0 sensoriales a largo plazo que, que al interactuar con diversas barreras, puedan impedir su participación plena y efectiva en la sociedad en igualdad de condiciones 
que las demás" (Ley 8661 Aprobación de la Convención de sobre los Derechos de las Personas con Discapacidad, Asamblea Legislativa de Costa Rica, 2008, p. 85).

- Género: construcción social de la diferencia sexual, que da lugar a la división jerárquica de la humanidad, mediante la atribución de características y roles diferenciados para hombres y mujeres, estableciendo relaciones de subordinación entre los sexos (Oliva, 2005).

- Perspectiva de género: Implica introducir en el análisis de las diferencias sociales entre hombres y mujeres, el efecto de las atribuciones, ideas, representaciones y prescripciones sociales que operan como norma de desigualdad en función del sexo (Delgado, 2015).

- Sexo: es una característica natural o biológica.

- Sexismo ambivalente: se estructura a través de la presencia de dos elementos con cargas afectivas antagónicas: positivas y negativas (Glick y Fiske, 1996; 2001). Lo cual se expresa en dos tipos de sexismo: el hostil y el benevolente.

- Sexismo hostil (SH): es el elemento que caracteriza a las mujeres como un grupo subordinado y legitima el control social que ejercen los hombres mediante actitudes tradicionales (paternalismo dominador, diferenciación de género competitiva, hostigamiento sexual).

- Sexismo benevolente (SB): es mucho más sutil; es el conjunto de actitudes interrelacionadas hacia las mujeres, de hecho sexistas en cuanto las consideran de forma estereotipada y limitadas a ciertos roles, aunque pueden tener un tono "afectivo", así como suscitar comportamientos típicamente categorizados como prosociales o de búsqueda de intimidad (Glick y Fiske, 1996, p. 491).

Como categorías previamente definidas especificamos las siguientes:

- Sentido de la diferencia: retomamos a Brah (2006) quien comprende que la diferencia se marca a partir de significados socialmente atribuidos a quienes se salen de la 'norma'. Esta autora acota cuatro acepciones, a saber: a) diferencia en términos de experiencia o proceso de significación de la realidad; b) diferencia como relación social; c) diferencia en términos vivenciales de la subjetividad individual y d) diferencia como identidad construida a partir de las anteriores. Lo diferente se vive como amenaza y por tanto se rechaza. 
- Sentido de no pertenencia: hacia las personas o grupos de personas con los cuales no nos identificamos, porque no pertenecemos a ese grupo externo (exogrupo); mecanismo de protección ante los grupos o integrantes de los grupos que nos resultan ajenos (Allport, 1971; Fiske, 1998).

- Discriminación positiva: medidas 'correctivas' y actuaciones tendientes a reducir las barreras que limitan el acceso y oportunidades de las PccD.

- Demandas excesivas: en relación con los prejuicios y el racismo simbólico (Henry y Sears, 2002).

- Poca familiaridad con PccD: la interacción con PccD genera familiaridad, empatía e identificación personal; no así la sola proximidad o cercanía.

- Desconocimiento y falta de información: la falta de información acerca de las PccD genera sentimientos negativos (temor, inseguridad, rechazo, incomodidad) que se expresan al tener un estudiante con condición de discapacidad en los cursos universitarios (Fontana, Quirós y Vargas, 2015).

- Sentimientos de lástima, compasión, admiración: la poca familiaridad con PccD genera sentimientos e idealizaciones sobre los y las estudiantes universitarios con discapacidad (Fontana, Quirós y Vargas, 2015) a quienes se les percibe como sujetos con poca autonomía e independencia.

- Efecto perceptual: al ver a una persona con alguna discapacidad, la atención se centra, perceptualmente, en la discapacidad y no en la persona, causando una actitud negativa que parte de la impresión visual (Wright, 1983).

Por último, la inclusión de una subescala de género permitió integrar en la escala la interseccionalidad entre el prejuicio hacia la discapacidad y el sexismo o prejuicio por razón de sexo, con el fin de investigar la doble discriminación de las mujeres con discapacidad, según refiere la literatura sobre el tema. La figura 1 resume el proceso descrito para la elaboración de los ítemes iniciales. 
Figura 1: Proceso para la delimitación del dominio del constructo a medir

Esquema para la elaboración de los ítemes iniciales de la Escala

\begin{tabular}{|c|c|}
\hline \multicolumn{2}{|c|}{ Premisa: problema o punto de partida } \\
\hline \multicolumn{2}{|c|}{ Constructo central: Prejuicios sutiles } \\
\hline \multicolumn{2}{|c|}{ Caracterización de los prejuicios sutiles hacia las PccD } \\
\hline Constructos de base & Categorías previas \\
\hline Discriminación & \\
\hline Discapacidad & Sentido de la diferencia \\
\hline $\begin{array}{l}\text { Personas con condición de discapacidad } \\
\text { (PccD) }\end{array}$ & Sentido de no pertenencia \\
\hline Género & Discriminación positiva \\
\hline Perspectiva de género & Demandas excesivas \\
\hline Sexo & Poca familiaridad con PccD \\
\hline Sexismo ambivalente & Desconocimiento y falta de información \\
\hline Sexismo hostil $(\mathrm{SH})$ & Sentimientos (lástima, compasión, admiración) \\
\hline Sexismo benevolente (SB) & Efecto perceptual \\
\hline
\end{tabular}

Fuente: Elaboración propia, 2017.

Cabe mencionar que para el primer diseño de la escala partimos, en primer lugar, de los resultados de la tesis de Vargas (2013) quien logra develar un conjunto de prejuicios sutiles a partir del análisis del discurso, dado que el análisis crítico del discurso en sus distintos niveles y dimensiones permitió identificar algunos de los prejuicios sutiles hacia las personas con discapacidad que prevalecen en la población académica universitaria, por medio de las estrategias discursivas utilizadas por las personas actuantes en el contexto institucional universitario, según se explica a continuación.

\subsection{Dimensiones, niveles y estrategias de valoración de los prejuicios sutiles}

En cada uno de los distintos niveles (retórico-argumentativo, semántico y pragmáticodiscursivo) se encuentran organizadas estrategias discursivas. Con respecto a las dimensiones del discurso, la dimensión práctica discursiva se basa en van Dijk (1996) para interpretar y develar algunos rasgos ideológicos utilizados por un colectivo o grupo social específico. En esta dimensión se han identificado algunas creencias que tienen las personas 
actuantes sobre las prácticas educativas y sociales hacia el estudiantado con discapacidad en el contexto institucional universitario de la Universidad Nacional de Costa Rica.

En la dimensión textual se seleccionaron tres niveles para el análisis discursivo con el propósito de develar los prejuicios: el retórico-argumentativo, el semántico y el pragmáticodiscursivo. Cada uno de ellos cuenta con sus estrategias discursivas características, según Vargas (2013). Estos tres niveles se utilizan en la definición constitutiva del constructo como se explica a continuación.

\subsection{Definición constitutiva del constructo: Prejuicio sutiles}

A partir del análisis del discurso como estrategia investigativa que posibilita indagar y develar los prejuicios hacia las personas con discapacidad, se planteó una definición del constructo partiendo de los niveles discursivos identificados como constitutivos del mismo, tal como se muestra en la Figura 2.

Figura 2: Definición del prejuicio sutil y niveles del discurso

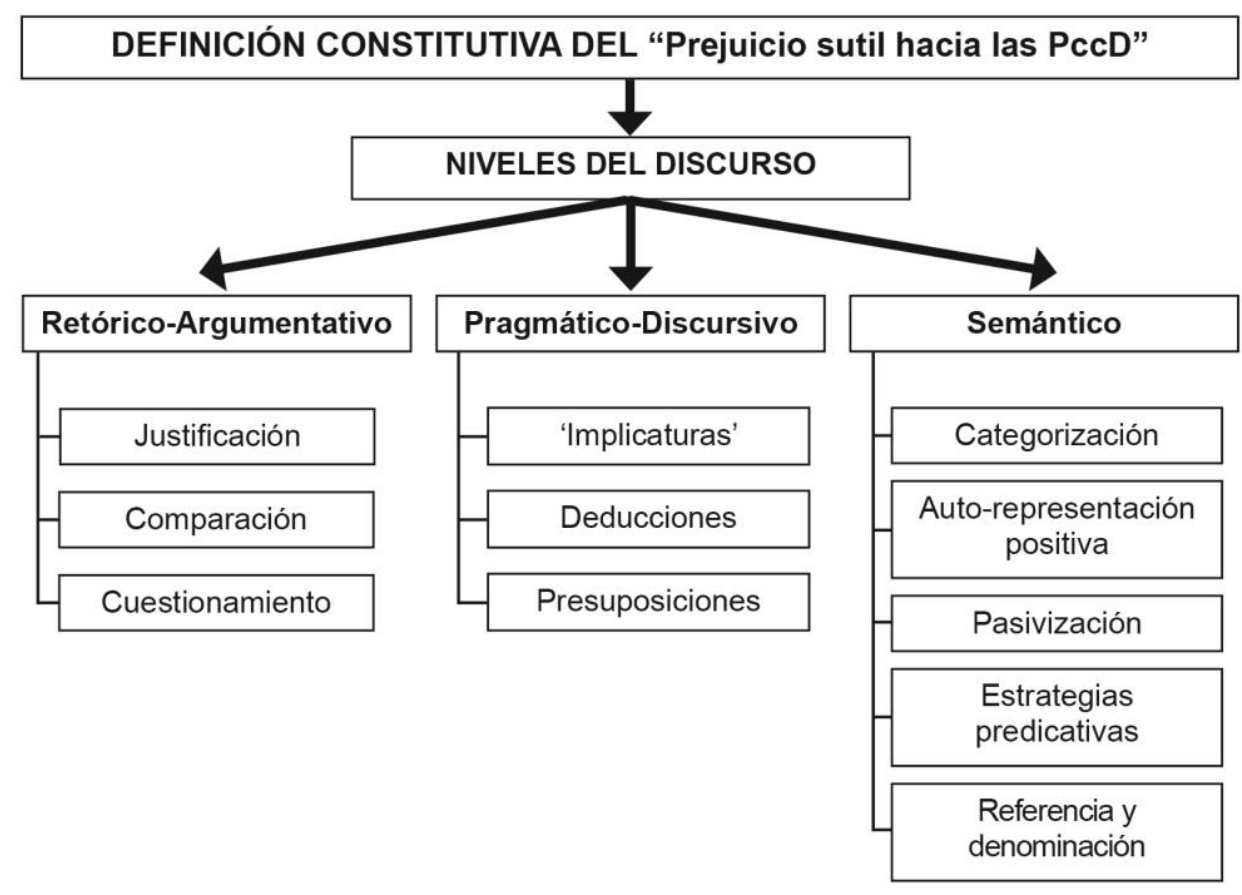

Fuente: Vargas, 2013 
Con el fin de establecer relaciones semánticas a partir de la definición constitutiva del constructo "prejuicio sutil hacia las Personas con condición de discapacidad" y derivar una definición operativa, formulamos indicadores (representados por Cn en la Figura 3), que permitirán redactar los ítemes (representado por C'n en dicha figura) según se muestra a continuación:

Figura 3: Relaciones semánticas e indicadores del prejuicio sutil.

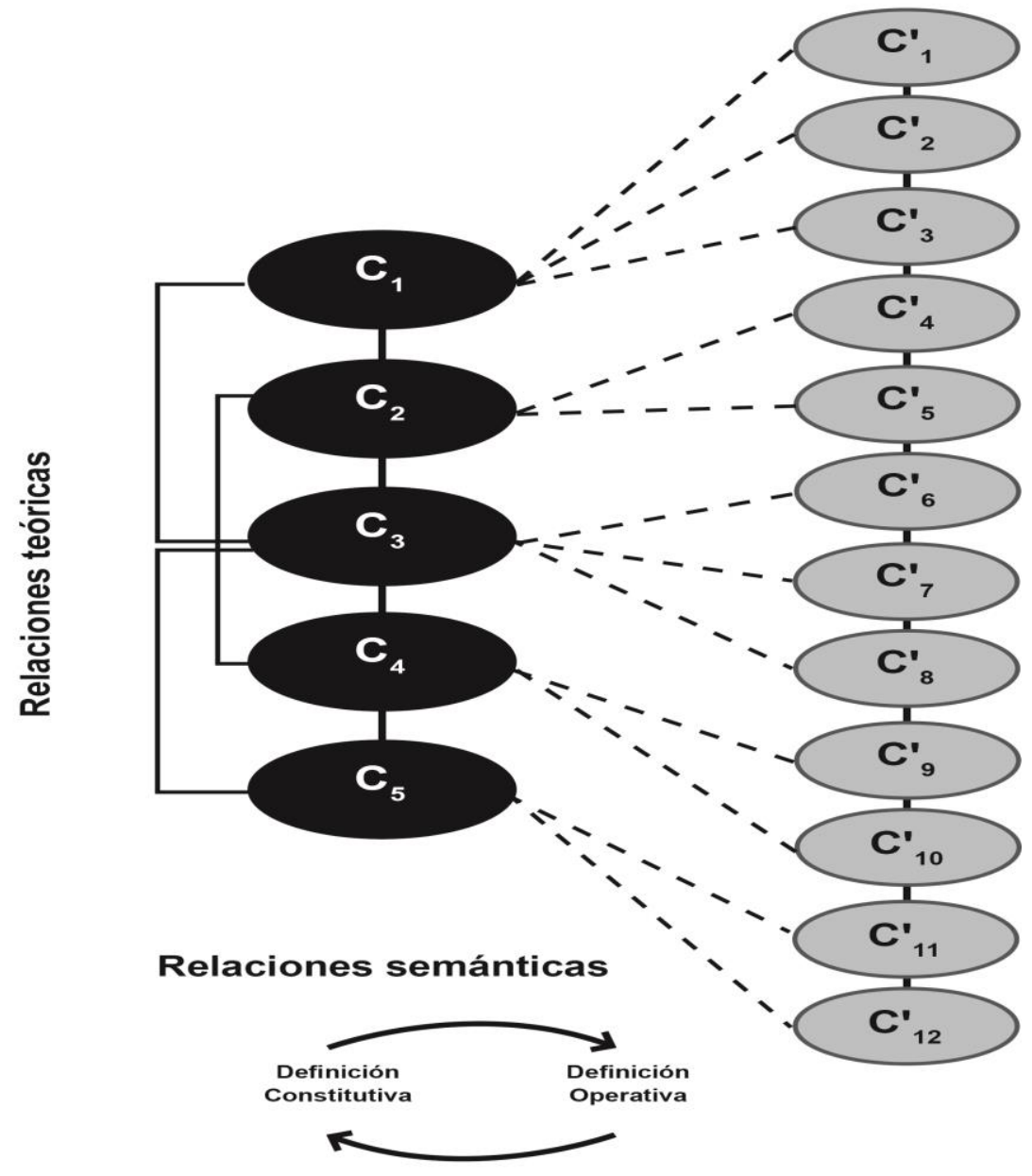

Fuente: Delgado, 2014

Sin duda esta fase es de mucha relevancia para el desarrollo de la investigación, pues de las relaciones teóricas y semánticas establecidas depende la consistencia constitutivaoperativa de la escala, razón por la cual, el tiempo invertido en esta fase fue alto y de mucho valor. 


\subsection{Argumentos para la discusión}

Un aspecto muy relevante consistió en incluir la dimensión de género, con el fin de valorar si el prejuicio hacia las personas con discapacidad es atravesado por el género; es decir, el modo en que opera de manera diferente en función del sexo de las personas con discapacidad. Por ello nos planteamos la posibilidad de hacerlo de dos formas, optando finalmente por la primera de ellas:

- Psicométrica: incluyendo una subescala específica de género dentro de la escala, formada por un conjunto de ítemes específicos para medir este aspecto. Se tomaron ítemes de escalas ya existentes de sexismo, adaptándolos a la discapacidad.

- Experimental: realizando estudios comparativos de las puntuaciones en la escala, manipulando el sexo como variable-estímulo.

Por lo tanto, este estudio si bien parte de un enfoque cualitativo y retoma los hallazgos de la investigación cualitativa desarrollada previamente en la tesis doctoral ya mencionada, para la elaboración de la escala el enfoque seguido fue prioritariamente de corte cuantitativo.

Para el diseño de la escala se optó por la técnica de auto-informe tipo Likert, útil para la medición de actitudes, en la que se presentan al sujeto una serie de afirmaciones (ítemes o reactivos) ante las cuales las personas manifiestan su grado de acuerdo o desacuerdo con cada enunciado, posicionándose en un rango de valoraciones posibles. Si bien la propuesta original de Likert ofrecía 5 puntos o alternativas de respuesta, la investigación y su posterior discusión, mostró que este escaso número de alternativas hace disminuir la fiabilidad, siendo más recomendable utilizar 7 puntos cuando el nivel cultural de la población objeto de estudio lo permite (Matas, 2018). Tratándose en nuestro caso de población universitaria, se utilizó por tanto el formato de 7 puntos: 1= Totalmente de Desacuerdo (TD), 2 = Bastante en Desacuerdo (BD), 3 = Ligeramente en Desacuerdo (LD), 4 = No sé, no puedo opinar (NP), 5= Ligeramente de Acuerdo (LA), 6 = Bastante de Acuerdo (BA) 7 = Totalmente de Acuerdo (TA). Otra ventaja de aumentar el número de alternativas de respuesta es que se incrementa la sensibilidad de la escala, lo que supone mayor precisión en medición, particularmente cuando se desee valorar intervenciones para modificar actitudes o prejuicios como es el caso (Bisquerra y Pérez-Escoda, 2015). También se valoraron ventajas e inconvenientes de que el número de opciones de respuesta fuera impar (7) o par (6), dado que algunos investigadores han introducido la práctica de eliminar el punto intermedio, con el fin de forzar el 
posicionamiento de los sujetos a favor o en contra. Dado que la eliminación de la opción intermedia no mejora las propiedades psicométricas de la escala, se optó por incluir la categoría intermedia ( 4 = ni acuerdo ni desacuerdo) por dos razones: para evitar la inducción de error en la respuesta al forzar artificiosamente el posicionamiento (Matas, 2018) y por una cuestión ética de respeto a las personas que prefieren no posicionarse.

Así pues, una vez definido el constructo a medir, en este caso el prejuicio hacia las personas con condición de discapacidad, se especificaron los indicadores. Los indicadores son los componentes o aspectos incluidos en la definición, que deben cumplir dos condiciones: pertinencia y exhaustividad. La pertinencia se refiere a su adecuación para medir el constructo tal como ha sido definido y la exhaustividad debe asegurar que el conjunto de indicadores cubre todos los aspectos relevantes del constructo (Delgado, 2014). A partir de los indicadores se procedió a la redacción de ítemes para cada uno de ellos, con el fin de obtener una batería amplia que permitiera someterlos a análisis de calidad psicométrica una vez aplicados a una muestra representativa de la población objeto de estudio.

El problema de fondo en toda escala de actitudes es, sin lugar a dudas, su validación o grado en que medimos lo que queremos medir, siendo la fiabilidad una condición previa y necesaria para la validez. Si bien los instrumentos de auto-informe, como la técnica Likert, presentan limitaciones (Delgado, Estrada y López, 2016) para medir cuestiones socialmente "sensibles" como el prejuicio, el rigor psicométrico en el proceso de elaboración permite controlarlas aceptablemente, por lo que la técnica Likert se sigue considerando un recurso útil, de amplia utilización en la investigación social (Matas, 2018).

En nuestro caso el proceso seguido para la elaboración y validación inicial de la escala según los Estándares de medición de la American Educational Research Association, el National Council on Measurement in Education y la American Psychological Association (AERA, NCME y APA, 2014) fue el siguiente: a) formulación de indicadores para cada una de las categorías propuestas en la definición constitutiva del constructo; b) revisión y validación de indicadores mediante consulta a jueces expertos; c ) construcción de los ítemes; d) obtención de evidencias de validez de contenido de los ítemes mediante consulta a jueces expertos; e) aplicación a una muestra piloto; e) depuración de la escala en función de la discriminación de los ítemes y la consistencia interna de la escala; y f) diseño definitivo de la 
escala para su aplicación a una muestra representativa de estudiantes y docentes de la UCR y la UNA.

Para la selección de participantes se utilizó un muestreo no probabilístico incidental, invitando a participar voluntariamente tanto a estudiantes como profesorado docente e investigador y personal de administración y servicios. Para la primera versión de la escala o prueba piloto se identificaron colegas del cuerpo docente de ambas instituciones, a quienes se les explicó su propósito y se les solicitó llevar la inquietud a los grupos que tienen a su cargo. Para la segunda versión o escala definitiva se utilizó, asimismo, una muestra no probabilística $^{3}$. Dado que el objetivo en esta fase de la investigación es evaluar la calidad psicométrica del instrumento, se trataría de un tipo de diseño "instrumental", para el cual la utilidad de la muestra no probabilística reside no tanto en la "representatividad", sino en una controlada elección de sujetos con características similares a la población objeto de estudio (Ato, López y Benavente, 2013; Montero y León, 2007; León y Montero, 2015).

A manera de reflexiones finales, cabe mencionar que la escala, además de contar con sus dimensiones teórico-epistemológica y metodológica, también cuenta con otras dos dimensiones: la política y la ética. Por esta razón hemos considerado relevante y urgente abrir el camino para identificar los prejuicios sutiles hacia las personas con condición de discapacidad, ya que se parte del supuesto de que no tenemos prejuicios hacia las otras personas. Sin embargo, no siempre somos conscientes de cuáles son nuestros prejuicios sutiles o latentes hacia las personas con condición de discapacidad, por lo que la escala explorará las dos dimensiones del prejuicio (hostil y benevolente) y la interacción con el prejuicio de género.

\section{Conclusiones}

Para la Educación Superior en Costa Rica, al igual que en otros países en los que se promueve la Educación Inclusiva, continúa siendo un reto responder adecuadamente al aumento de la población con discapacidad que ingresa a las instituciones universitarias

\footnotetext{
3Un abordaje exhaustivo del proceso metodológico de construcción de la escala superaría los límites de este artículo sobre la fundamentación teórica y el diseño de la escala. Por esta razón, dicho proceso y los resultados de la validación psicométrica según los Standards for Psychological and Educational Testing (AERA, NCME \& APA, 2014) serán analizados y discutidos en un próximo artículo.
} 
(Gurdián y Vargas, 2018), así como superar actitudes y prejuicios discriminatorios dentro de las aulas en el ámbito universitario (Gurdián, 2002).

En muchos ámbitos se maneja un discurso idealista y "políticamente correcto" sobre los derechos humanos y de la diversidad, que nos devuelve una imagen idealizada de nosotros mismos como seres humanos y solidarios porque aceptamos la diversidad. Esta imagen, más que un reflejo de la adecuación de nuestras prácticas inclusivas, es un reflejo de las buenas intenciones con que quisiéramos afrontarlas. Pero la aceptación del discurso no produce todas las acciones requeridas para identificar los prejuicios que hemos interiorizado en una cultura de la desigualdad y, menos aún, para superarlos. Los prejuicios como tales, no se han abordado suficientemente o, por lo menos, no de forma adecuada, pues siguen prevaleciendo y afectando la vida de muchas personas incluso por parte de personas bien intencionadas que ni siquiera los han identificado en ellas mismas, o que queriendo compensar sus efectos nocivos adoptan formas benevolentes que en realidad los reproducen y afianzan. Sus repercusiones continúan concretándose en prácticas excluyentes y discriminatorias. Pareciera que aún no es mucho lo que se hace por reconocerlos, trabajarlos y tratar de superarlos.

La inclusión educativa y social de las personas con discapacidad con mucha frecuencia se reduce a la valoración de aspectos externos y visibles, tales como la eliminación de barreras arquitectónicas: aceras y callesin en mal estado, ausencia de rampas, falta de espacios públicos accesibles, falta de recursos o rehabilitación, entre otras. Sin embargo, las barreras más importantes para la participación, inclusión y desarrollo de las personas con discapacidad son de origen actitudinal: los prejuicios, la exclusión y la discriminación que la "cultura de la productividad" inscribe en nuestras percepciones y valoraciones de los demás, sin que ni siquiera seamos conscientes de ello. La diversidad funcional de las personas se interioriza como discapacidad, minusvalía y déficit para tareas concretas, generalizando el rasgo limitante específico de la persona, a la persona en su totalidad. La limitación respecto a una "tarea específica" se significa como como rasgo sustantivo que minusvalora la totalidad de la persona.

El prejuicio aún cuando es una construcción social, parece ser inherente al ser humano: tendemos a prejuzgar. Consciente o inconscientemente generamos, reproducimos o repetimos algún prejuicio. El punto álgido son las consecuencias sociales negativas que genera, ya que con ellas el pensamiento se plasma en acciones concretas, convirtiendo lo 
que debería ser un trato diferenciado para tareas específicas afectadas por la diversidad funcional en un trato desigual de la persona y obteniendo como resultado la discriminación. Es importante recordar que los prejuicios son las ideas preconcebidas que tenemos sobre las otras personas sin apenas conocerlas; son nuestra forma de encasillarlas por distintas razones: la educación recibida, nuestras propias experiencias o vivencias en el ámbito familiar o escolar, en el barrio o comunidad a la que pertenecemos y, en último término, en la cultura en la que nos socializamos.

Desde este compromiso ético-político de la investigación, que tiene como uno de sus objetivos la promoción de la igualdad para todas las personas sin discriminación por razón de algún tipo de diversidad, la escala construida cobra relevancia, por ejemplo, para un plan de investigación diagnóstica o evaluativa sobre la educación inclusiva. Un instrumento de evaluación con garantías de fiabilidad y evidencias de validez, que permita identificar qué prejuicios perviven en las prácticas educativas a pesar de las mejores intenciones de quienes los reproducen, será muy útil para emprender prácticas inclusivas adecuadas. Así, por ejemplo, puede servir para emplearse en diversas actividades y programas formativos docentes, sesiones de capacitación, información, actualización y concienciación en el abordaje de la temática de discapacidad en los contextos de educación superior, ante la prevalencia de prejuicios hacia las personas con discapacidad. Puesto que muchos de los prejuicios como tales se derivan del temor e incertidumbre, de la falta de formación y actualización que causa no saber cómo atender a un estudiante con discapacidad en los cursos que se imparten, identificarlos y hacerlos conscientes permitirá hacer un diagnóstico de necesidades. Sostenemos que "todo saber pasa por la subjetividad" (Gurdián, 2011, p. 6); en consecuencia, para que se produzca un cambio en la realidad universitaria que conocemos, debe efectuarse un cambio en el plano subjetivo y ese cambio se debe dar desde la intersubjetividad. Por tanto, el cambio en las construcciones sociales, los sentidos y los significados acerca de la discapacidad, es un cambio urgente y necesario que debe darse desde la diada subjetividad-intersubjetividad.

\section{Referencias}

AERA, NCME \& APA. (2014). Standards for Educational and Psychological Testing. Washington, D.C.: American Educational Research Association.

Allport, Gordon. (1971). La naturaleza del prejuicio. Buenos Aires, Argentina: EUDEBA. 
Amorós, Celia. (2005). La gran diferencia y sus pequeñas consecuencias para las luchas de las mujeres. Madrid: Cátedra.

Asamblea Legislativa de Costa Rica. (2008). Ley 8661 Aprobación de la Convención de sobre los Derechos de las Personas con Discapacidad. San José, Costa Rica: CONAPDIS.

Ato, Manuel; López, Juan J. y Benavente, Ana (2013). Un sistema de clasificación de los diseños de investigación en psicología. Anales de Psicología, 29(3), 1038-1059.

Bisquerra, Rafael y Pérez-Escoda, Nuria. (2015). ¿Pueden las escalas Likert aumentar la sensibilidad? REIRE, 8(2), 129-147.

Brah, Avtar. (2006). Diferencia, diversidad y diferenciación. Cuadernos Pagu, 26, 1-33.

Delgado, Carmen. (2014). Viajando a Ítaca por mares cuantitativos. Manual de ruta para investigar en grado y postgrado. Salamanca: Amarú.

Delgado, Carmen. (2015). Por qué la violencia de género es algo diferente. Reenfocando el concepto. En A. Figueruelo, M. del Pozo y M. León (Coord.), ¿Podemos erradicar la violencia de género? Análisis, debate y propuestas (pp. 41-56). Granada: Comares.

Delgado, Carmen; Estrada, Benito, y López, José Alfredo. (2016). Limitaciones de los autoinformes en la evaluación de la violencia en la pareja. Infocop Online. Recuperado de http://www.infocop.es/view article.asp?id=5998

Fairclough, Norman. (1992). Discourse and social change. Cambridge: Polity Press.

Ferrer, Victoria y Bosch, Esperanza. (2005). Introduciendo la perspectiva de género en la investigación psicológica sobre violencia de género. Anales de Psicología, 21,1, 1-10

Fiske, Susan. (1998). Stereotyping, prejudice and discrimination. En D. T. Gilbert, S. T. Fiske, \& G. Lindzey (Eds.), Handbook of social psychology (4th ed., Vol. 2, pp. 357-414). New York: McGraw-Hill.

Fontana, Angélica; Quirós, Marco y Vargas, Marie-Claire. (2015). Construcciones sobre discapacidad y necesidades educativas e implicaciones en la respuesta educativa del estudiantado de la Universidad Nacional, Costa Rica (UNA). Ponencia presentada en las IX Jornadas Científicas de Investigación sobre Personas con Discapacidad, Instituto Universitario de Integración en la Comunidad (INICO), Universidad de Salamanca. Marzo, 2015. 
García, Juan José y Zarco, Juan. (2004). El espejo social de la mujer con gran discapacidad. Madrid: Fundamentos.

Glick, Peter \& Fiske, S. T. (1996).The Ambivalent Sexism Inventory: Differentiating Hostile and Benevolent Sexism. Journal of Personality and Social Psychology, 70, 491-512.

Glick, Peter \& Fiske, Susan. (2001). An ambivalent alliance: Hostile and benevolent sexism as complementary justifications for gender inequality. American Psychologist, 56(2), 109-118.

Gurdián, Alicia. (2002). Rompiendo prejuicios y provocando rupturas. En R. Jiménez (Ed.), Las personas con discapacidad en la Educación Superior. (p. 127-139). San José, Costa Rica: Fundación Justicia y Género.

Gurdián, Alicia. (2010). El Paradigma Cualitativo en la Investigación Socio-Educativa.San José, Costa Rica: Editorial de la Universidad de Costa Rica.

Gurdián, Alicia y Vargas, Marie-Claire. (2017). Informe parcial Proyecto Construyendo una escala de prejuicios hacia las personas con discapacidad. San José/Heredia: INIE, UCR-CIDE, UNA.

Hanafin, Joan; Shevlin, Michael.; Kenny, Mairim \& Mc Neela, Eileen. (2009). Including Young people with disabilities; Assessment challenges in higher education. HigherEducation, $54,435-448$.

Harding, Sandra. (1996). Ciencia y feminismo. Madrid: Morata.

Henry, PJ \& Sears, David. (2002).The Symbolic Racism 2000 Scale. Political Psychology, 23(2), 253-283.

Iñiguez, Lupicinio. (2006). Análisis del discurso. Manual para las ciencias sociales (2ª ed.). Barcelona: Editorial UOC.

Lissi, Rosa; Zuzulich, Soledad; Salinas, Marcela; Achiardi, Consuelo; Hojas, Ana María y Pedrals, Nuria (2009). Discapacidad en contextos universitarios: Experiencia del PIANE UC en la Pontificia Universidad Católica de Chile. Calidad en la Educación, (30), 305324.

López, María. (2007). Discapacidad y género. Estudio etnográfico sobre mujeres con discapacidad. Educación y diversidad. Revista inter-universitaria de investigación sobre discapacidad e interculturalidad, 1, 137-172 
León, Orfelio y Montero, Ignacio. (2015). Métodos de investigación en Psicología y Educación. La tradición cuantitativa y cualitativa (4⿳亠丷⿵冂丶 ed.). Madrid, España: McGraw-Hill Educación.

Marks, Deborah. (1999).Disability: Controversial debates and psychosocial perspectives. Londres: Routledge.

Matas, Antonio. (2018). Diseño del formato de escalas tipo Likert: un estado de la cuestión. Revista Electrónica de Investigación Educativa, 20(1), 38-47.

Mohanty, Chandra Talpade (2011). De vuelta a "Bajo los ojos de Occidente": La solidaridad feminista a través de las luchas anticapitalistas. En L. Suárez y R.A. Hernández, Descolonizando el feminismo, Teorías y prácticas desde los márgenes (pp. 407-464). Madrid: Ediciones Cátedra.

Montero, Ignacio y León, Orfelio (2007). A guide for naming research studies in psychology. International Journal of Clinical and Health Psychology, 7(3), 847-862.

Montes, Beatriz. (2008). Discriminación, prejuicio, estereotipos: conceptos fundamentales, historia de su estudio y el sexismo como nueva forma de prejuicio. En: Iniciación a la Investigación Revista Electrónica Universidad de Jaén. Inilnv, 3: a1

Morales, Esperanza. (2004). Las aproximaciones americanas al análisis del discurso oral: perspectivas de futuro. En D. Pujante (Coord.), Caminos de la Semiótica en la última década del siglo XX (pp. 109-133) Valladolid, España: Universidad de Valladolid.

Oliva, Asunción. (2005). Debates sobre el género. En C. Amorós y A. de Miguel, Teoría feminista: de la ilustración a la globalización (pp. 15-60). Madrid. España: Editorial Minerva.

Pettigrew, Thomas \& Meertens, Roel. (1997).Is subtle prejudice really prejudice? Public Opinion Quaterly, 61, 54-71.

Pettigrew, Thomas \& Meertens, Roel. (2001). In defense of the subtle prejudice concept: a retort. European Journal of Social Psychology, 31, 299-309.

Priestley, Mark. (1998). Constructions and Creations: idealism, materialism and disability theory, Disability \& Society, 13(1), 75-94.

Ramírez-Morera, Marcela. (2015). La inclusión del género y la discapacidad en el currículum de la Escuela de Trabajo Social, Sede Rodrigo Facio, Universidad de Costa Rica. Revista Reflexiones, 94(1), 49-63

Saltzman, Jane. (1992). Equidad y género. Una teoría integradora de estabilidad y cambio. Madrid: Cátedra. 
Suárez, Liliana (2011). Colonialismo, gobernabilidad y feminismos poscoloniales. En L.

Suárez y R.A. Hernández, Descolonizando el feminismo, Teorías y prácticas desde los márgenes (pp. 31-73). Madrid: Ediciones Cátedra.

van Dijk, Teun. (1983). The cognitive representation of attitudes and prejudice. Forum Linguisticum, 7(3), 189-204.

van Dijk, Teun. (1984). Prejudice in discourse. Amsterdam: John Benjamin Publising Co.

van Dijk, Teun. (1996). Análisis del discurso ideológico [Versión6, pp. 15-43]. Recuperado de http://www.google.co.cr/\#hl=es\&rlz=1R2WZPC esCR376\&sclient=psyab\&q=versi\%C3\%B3n+6+UAM+M\%C3\%A9xico\&rlz=1R2WZPC esCR376\&oq=versi\%

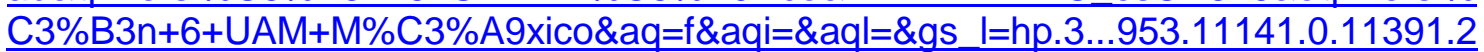
3.21.1.0.0.0.688.7985.25j7j4j4.20.0...0.0.5fOvPYHftBw\&pbx=1\&bav=on.2,or.r gc.r pw. $\underline{r}$ qf.,cf.osb\&fp=8d279b53965541ec\&biw=1151\&bih $=522$

Vargas, Marie-Claire. (2013). Influencia de los prejuicios de un sector de la población Universitaria respecto a la discapacidad en la construcción de una cultura institucional inclusiva. Universidad Nacional. Costa Rica, 2012-2013 (Tesis Doctoral). Universidad de Costa Rica, Sede Rodrigo Facio, San José, Costa Rica.

Wright, Beatrice. (1983). Physical Disability-A Psychosocial Approach. NY: Harper \& Row Publishers. 
Revista indizada en

sciplo

redalyc. latindex

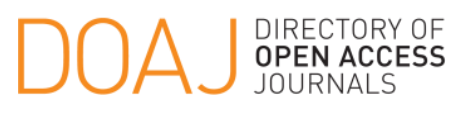

Distribuida en las bases de datos:

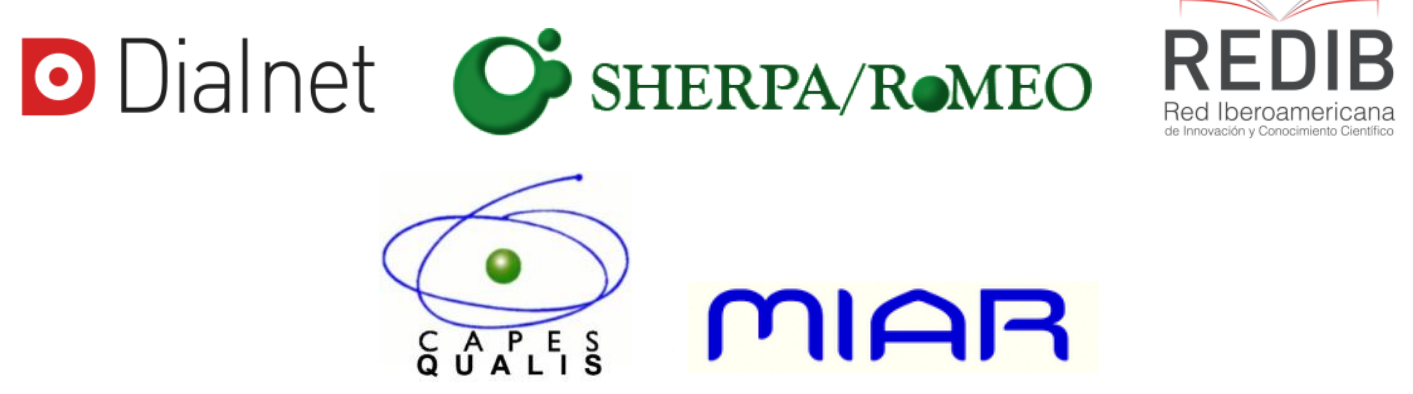

